International Journal of Current Advanced Research

ISSN: O: 2319-6475, ISSN: P: 2319 - 6505, Impact Factor: SJIF: 5.995

Available Online at www.journalijcar.org

Volume 6; Issue 5; May 2017; Page No. 3623-3627

DOI: http://dx.doi.org/10.24327/ijcar.2017.3627.0333

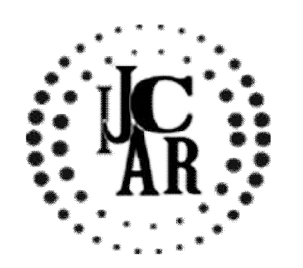

Research Article

\title{
GLOBAL INDUSTRIAL PROCESS MONITORING USING RASPBERRY PI CONTROLLER
}

\author{
Sayali Gajanan Desai., B. T. Salokhe and A. S. Mali
}

Department of Electronics, T.K.I.E.T Warananagar

\begin{tabular}{l}
\hline A R T I C L E I N F O \\
\hline Article History: \\
Received $7^{\text {th }}$ February, 2017 \\
Received in revised form $12^{\text {th }}$ March, 2017 \\
Accepted $15^{\text {th }}$ April, 2017 \\
Published online $28^{\text {th }}$ May, 2017 \\
\end{tabular}

Key words:

Raspberry pi; Wi-Fi module

\begin{abstract}
A B S T R A C T
This project proposes an advanced system for process management via a credit card sized single board computer called raspberry pi based multi parameter monitoring hardware system designed using RS232 and microcontroller that measures and controls various global parameters. The entire system is designed with the double layer SMD based embedded board with different sensors and a raspberry pi that can compile and communicate the data received from the sensors. The raspberry pi when operated on the Linux operating system can perform multi-tasking. There are varieties of methods to monitor the data remotely obtained by various sensors. But there is need of robust and easy to use computerized system. As computerized system involves high implementation costs, there is need of alternative solution in terms of equal robustness and cost effective.
\end{abstract}

Copyright $₫ 2017$ Sayali Gajanan Desai et al. This is an open access article distributed under the Creative Commons Attribution License, which permits unrestricted use, distribution, and reproduction in any medium, provided the original work is properly cited.

\section{INTRODUCTION}

This project proposes an advanced system for process management via This project a credit card sized single board computer called raspberry can be used as front end panel for monitoring and control. The java servers used to control the fast developing technology that connects all devices with internet [5]. For soft real time systems TCP, UDP and IP protocols are efficient [3] Embedded web server and Linux based system is cost effective with high performance [3]. The RS232 protocol is sufficient for parameter monitoring and control [2]. A printed circuit board (PCB) uses conductive tracks, pads and other features etched from copper sheets to connect the electronic components laminated onto a non-conductive substrate. Surface - mount technology (SMT) is a technique where the

Components are placed directly onto the surface of printed circuit boards (PCBs) Both technologies can be used in a combination i.e. the components that cannot be mounted can be used with through hole technology. In industrial automation, there are different manufactures producing their own PLCs [3]. The PLCs in an industry is connected with distributed control system (DCS) by protocols such as RS232/485, USB and Ethernet [1] [5]. The DCS has multilevel hierarchical network structure for communication. Due

*Corresponding author: Sayali Gajanan Desai

Department of Electronics, T.K.I.E.T Warananagar to the hierarchical network structure the communication becomes complex and high in cost. Complete network from field level to control level is not formed. The java simulators can be used as front end panel for monitoring and control. The java servers used to control the process in a field [5]. Internet of Things (IoT) is a fast developing technology that connects all devices with Internet [6].

There are varieties of methods to monitor the data remotely obtained by various sensors. But there is need of robust and easy to use computerized system. As computerized system involves high implementation costs, there is need of alternative solution in terms of equal robustness and cost effective To develop the robust remote data monitoring system we propose the system with following sub objectives

1. To develop the embedded system along with various sensors to measure voltage, current, fluid level, light intensity, temperature, humidity in industrial environment and send this data over Wi-Fi (2.4 $\mathrm{GHz}$ )module to remote receiver.

2. To develop the system with Raspberry pi module (2/3) to receive data from $\mathrm{Wi}-\mathrm{Fi}$ module $(2.4 \mathrm{GHz})$ and display it on monitor.

\section{System Discription}

To develop the robust remote data monitoring system we propose the system with following sub objectives. To develop the embedded system along with various sensors to measure voltage, current, fluid level, light intensity, temperature, humidity in industrial environment and send this data over Wi-Fi $(2.4 \mathrm{GHz})$ module to remote receive and To develop the 
system with Raspberry pi module(2/3) to receive data from Wi-Fi module $(2.4 \mathrm{GHz})$ and display it on monitor

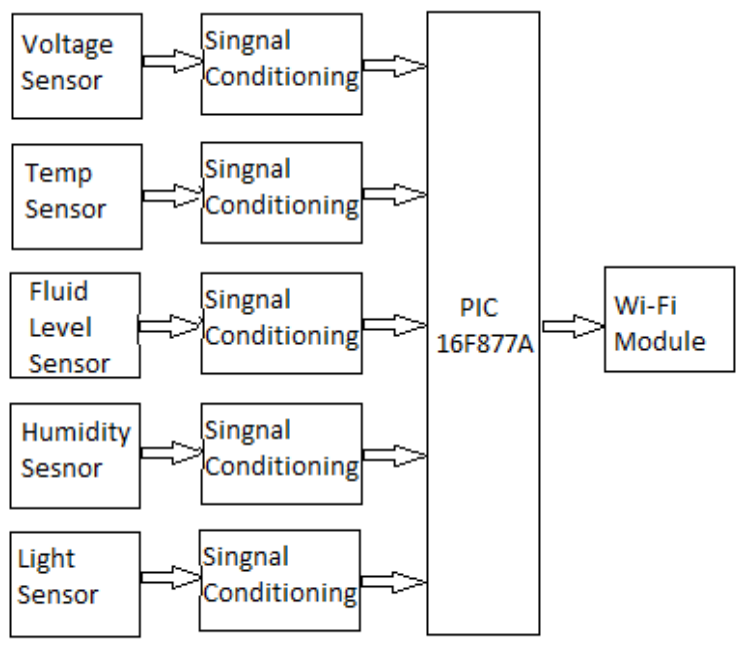

Fig 1 : Proposed System block Diagram For sensor Interface

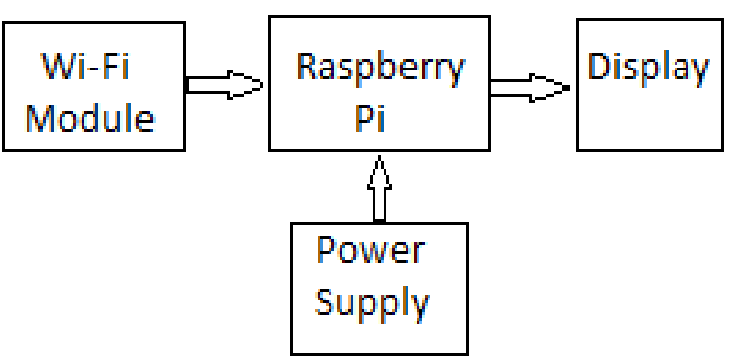

\section{Fig 2 : Propsed System Block Diagram For Moniotoring}

\section{Data Gathering}

Voltage, fluid level, humidity, temperature and light intensity can measured using different sensors and interfaced with PIC16F877A microcontroller. The in-built ADC can convert analog output of sensor to digital. This Digitized data can be sent to raspberry pi board using Wi-Fi module.

Raspberry pi based application can be developed which can receive the data from $\mathrm{Wi}-\mathrm{Fi}$ module. The display connected to raspberry pi can be PC monitor which will show the data.

\section{Temperature Sensor}

LM35 or RTD can be used to measure the environmental or specific process temperature as per specific industrial need. The OPAMP based signal conditioning circuit is required to calibrate the output of sensor with respect to desired input of ADC.

\section{Humidity Sensor}

SyHS220 sensor can be used to measure relative humidity in the environment. The output of this sensor is analog and can be calibrated using OPAMP based signal conditioning circuit.

\section{Fluid Level Sensor}

Hall effect based or potentiometer based level sensor can be used to measure fluid level. The analog output of the sensor can be calibrated using signal conditioning

\section{Voltage or current Sensor}

To monitor voltage level, simple step down transformer and half rectifier circuit can be used which varies in accordance with voltage change. To measure current, current transformer can be used which gives output in terms of voltage with respect to current flowing. The signal conditioning can be used to calibrate the output of the sensor.

\section{Light intensity sensor}

To measure the light intensity, the LDR based circuit can be used. The output of this circuit varies in accordance with change in light intensity falling on LDR sensor. The analog output can be calibrated using signal conditioning circuit.

\section{PIC16F877A}

The data gathered from different sensors is send to the signal conditioners to enhance its strength then all the data is gathered by PIC16F877A and is transmitted over Wi-Fi module to the Raspberry PI controller. And display it on monitor.

\section{Software Specification}

The following software tools are required for Designing, compiling and debugging

\section{MikroC Pro for PIC}

MikroC PRO is an ANSI C compiler for PIC devices of Microchip. The main features are intuitive IDE, Powerful compiler with advanced optimizations, lots of hardware and software library. Some of the tools that is integrated with this compiler are active comment editor, ASCII chart, EEPROM editor, GLCD bitmap editor, HID terminal, LCD custom character, Mikro boot loader, UDP terminal and USART terminal. Software simulator simulates the code flow in PC and supports all debugging modes MikroICD.

\section{Raspbian operating system}

Raspbian is a free operating system based on debian, Optimized for the raspberry pi hardware. Raspbian comes with over 35,000 packages and pre-compiled software bundled in a nice format for easy installation on Raspberry Pi. Raspbian is still under development to improve stability and performance of as many Debian packages as possible.

\section{Hardware Specification \\ Raspberry Pi Model B+}

Raspberry pi is based on the Broadcom BCM2835system on a chip (SoC) that includes an ARM1176JZF-S $700 \mathrm{MHz}$ processor, Video Core 4 GPU, and was originally designed with 256 megabytes of RAM and later upgraded to $512 \mathrm{MB}$. The system has either Secure Digital (SD) or MicroSD sockets for boot media and persistent storage. The other features of raspberry pi model $\mathrm{B}+$ are $700 \mathrm{MHz}$ cloak speed, four individual USB host ports, 10/100 Base T Ethernet port and HDMI audio and video output .

\section{PIC16F877A}

10-bit, up to 8-channel Analog-to-Digital Converter (A/D)

- Brown-out Reset (BOR)

- Analog Comparator module with:

- Two analog comparators 
- Programmable on-chip voltage reference (VREF) module

- Programmable input multiplexing from device inputs and internal voltage reference

- Comparator outputs are externally accessible

\section{Wi-Fi dongle with Raspberry Pi}

Wi-Fi is a popular wireless networking technology thus uses radio waves to provide wireless high-speed Internet and network connections. The Wi-Fi Alliance, the organization that owns the Wi-Fi registered trademark term specifically defines Wi-Fi as any "wireless local area network (WLAN) products that are based on the Institute of Electrical and Electronics Engineers' (IEEE) 802.11 standards. We are using Wi-Fi dongle is shown in Fig.6 for Wi-Fi communication between Raspberry Pi and clients. The first task is to bum the Raspbian OS into the raspberry pi and we can configure the device with following command then we can see a window as shown in Fig.. We need to swap "YOUR_SSID" our Wi-Fi network. Then reboot the raspberry pi using following command Sudo reboot. Now we are ready to use Wi-Fi communication. Raspberry Pi 3 has inbuilt Wi-Fi module which can also be used in our project.

\section{Hardware Discription}

\section{Temperature Sensor}

\section{LM35 Precision Centigrade Temperature Sensor}

The LM35 series are precision Integrated circuit temperature sensors whose output voltage is linearly proportional to the Celsius temperature. The LM 35 thus has an advantage over linear temperature sensors calibrated in Kelvin as the user is not required to subtract a large constant voltage from its output to obtain convenient centigrade scaling. The LM 35 does not require any external calibration or trimming to provide typical accuracies of $+/-(1 / 4)$ degree Celsius at room temp and $+/-(3 / 4)$ degree Celsius over a full -55 to +150 degree Celsius temp range.

\section{Humidity Sensor (SY-HS-220)}

Humidity Sensor detects the relative humidity of the immediate environments in which they are placed.

\section{Working Principle}

Most humidity sensors use capacitive measurement to determine the amount of moisture in the air .this type of measure relies on two electrical conductors with nonconductive polymer film laying between them to create an electrical field between them .moisture from the air collects on the film and cause changes in the voltage levels between the two plates this change is converted into a digital measurement.

\section{Liquid Level Sensor}

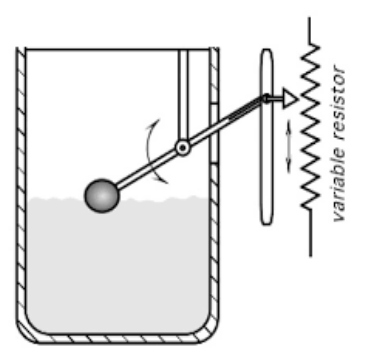

Fig 3 Liquid level sensor
The measurement of position, displacement or level is very essential for many vivid applications such as process feedback control, transportation traffic control, robotics, security systems and more. Here term position refers to determination of object's co-ordinates (either linear or angular) with respect to a selected reference. The term displacement refers to moving from one position to another position for a specific distance or angle.

A proximity sensor is a threshold version of a position detector. A position sensor is a linear device whose output signal represents a distance to the object from a certain reference point. A proximity sensor is a simpler device which generates the output signal when a certain distance to the object becomes essential for an indication. A displacement sensor often is part of a more complex sensor where the detection of movement is one of several steps in a signal conversion. An example is a pressure sensor where pressure is translated into a displacement of a diaphragm. Later the diaphragm displacement is converted into an electrical signal which represents pressure. Hence the position sensors are essential for the design of many other sensors. Position and displacement sensors are static devices whose speed response usually is not critical for the performance. Following specifications need to be considered while selecting or designing the displacement or position sensors.

\section{Voltage Sensor}

This module allows to measure voltages of $0-25 \mathrm{~V}$ by presenting a lower voltage to the output for measuring. After having this value, simply through some math actual voltage can be calculated. Plug + into $5 \mathrm{~V}$, - ground and $\mathrm{S}$ into an analogue pin.

\section{Current Sensor using current transformer}

Current transformers reduce high voltage currents to a much lower value and provide a convenient way of safely monitoring the actual electrical current flowing in an AC transmission line using a standard ammeter. The principal of operation of a basic current transformer is slightly different from that of an ordinary voltage transformer. Unlike the voltage or power transformer looked at previously, the current transformer consists of only one or very few turns as its primary winding. This primary winding can be of either a single flat turn, a coil of heavy duty wire wrapped around the core or just a conductor or bus bar placed through a central hole. Due to this type of arrangement, the current transformer is often referred to as a "series transformer" as the primary winding, which never has more than a very few turns, is in series with the current carrying conductor supplying a load. The secondary winding however, may have a large number of coil turns wound on a laminated core of low-loss magnetic material. This core has a large cross-sectional area so that the magnetic flux density created is low using much smaller cross-sectional area wire, depending upon how much the current must be stepped down as it tries to output a constant current, independent of the connected load.

The secondary winding will supply a current into either a short circuit, in the form of an ammeter, or into a resistive load until the voltage induced in the secondary is big enough to saturate the core or cause failure from excessive voltage breakdown. 
Unlike a voltage transformer, the primary current of a current transformer is not dependent of the secondary load current but instead is controlled by an external load. The secondary current is usually rated at a standard 1 Ampere or 5 Amperes for larger primary

Current transformers can reduce or "step-down" current levels from thousands of amperes down to a standard output of a known ratio to either $5 \mathrm{Amps}$ or $1 \mathrm{Amp}$ for normal operation. Thus, small and accurate instruments and control devices can be used with CT's because they are insulated away from any high-voltage power lines. There are a variety of metering applications and uses for current transformers such as with Wattmeter's, power factor meters, watt-hour meters, protective relays, or as trip coils in magnetic circuit breakers, or MCB's secondary windings are related by an inverse proportion. But relatively large changes in a current transformers turns ratio can be achieved by modifying the primary turns through the CT's window where one primary turn is equal to one pass and more than one pass through the window results in the electrical ratio being modified.

\section{Wi-Fi dongle with Raspberry Pi}

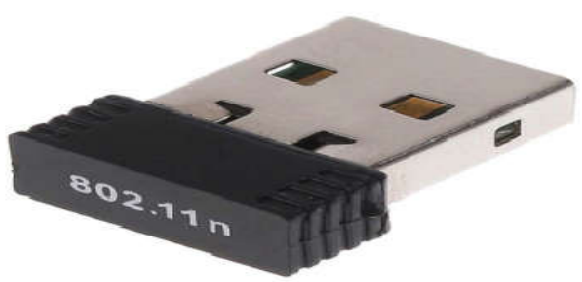

Fig 4 USB Wi-Fi Dongle for Raspberry pi

Wi-Fi is a popular wireless networking technology thus uses radio waves to provide wireless high-speed Internet and network connections. The Wi-Fi Alliance, the organization that owns the Wi-Fi registered trademark term specifically defines Wi-Fi as any "wireless local area network (WLAN) products that are based on the Institute of Electrical and Electronics Engineers' (IEEE) 802.11 standards. We are using Wi-Fi dongle is shown in Fig.6 for Wi-Fi communication between Raspberry Pi and clients. Raspberry Pi 3 has inbuilt Wi-Fi module which can also be used in our project.

\section{Raspberry Pi 3}

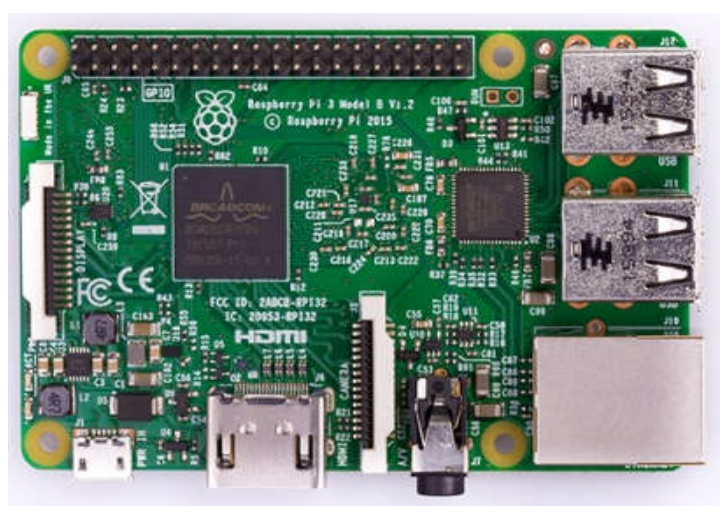

Fig 5 Raspberry Pi Controller

The Raspberry Pi 3 is the third generation Raspberry Pi. it has:

- A 1.2GHz 64-bit quad-core ARMv8 CPU
- $\quad 802.11 n$ Wireless LAN

- Bluetooth 4.1

- Bluetooth Low Energy (BLE)

- $1 \mathrm{~GB}$ RAM

- Video Core IV 3D graph

The Raspberry Pi 3 has an identical form factor to the previous $\mathrm{Pi} 2$ (and $\mathrm{Pi} 1$ Model $\mathrm{B}+$ ) and has complete compatibility with Raspberry Pi 1 and 2.

\section{Serial Wi-Fi Module for PIC side}

\section{ModuleESP8266}

ESP8266 Wi-Fi Module is a self contained SOC with integrated TCP/IP protocol stack that can give any microcontroller access to your Wi-Fi network. The ESP8266 is capable of either hosting an application or offloading all Wi-Fi networking functions from another application processor. Each ESP8266 module comes pre-programmed with an AT command set firmware, meaning, you can simply hook this up to your Adriano device and get about as much Wi-Fi-ability as a Wi-Fi Shield offers (and that's just out of the box)! The ESP8266 module is an extremely cost effective board with a huge and ever growing community. This module has a powerful enough on-board processing and storage capability that allows it to be integrated with the sensors and other application specific devices through its GPIOs with minimal development up-front and minimal loading during runtime. Its high degree of on-chip integration allows for minimal external circuitry, including the front-end module, is designed to occupy minimal PCB area. The ESP8266 supports APSD for VoIP applications and Bluetooth coexistence interfaces. It contains a self-calibrated RF allowing it to work under all operating conditions, and requires no external RF parts.

\section{Future Work}

The system can be enhanced for wave form presentation of data in an excel sheet using raspberry pi. Controlling actions can be set for some predefined cases which enables the automatic operation at certain cases. a dedicated video processor can be used in raspberry pi to display graphical and three dimensional view of the industry.

\section{CONCLUSION}

There are varieties of methods to monitor the data remotely obtained by various sensors. But there is need of robust and easy to use computerised system. As computerised system involves high implementation cost, there is need of alternative solution in terms of equal robustness and cost effective. This paper gives the solution by using a raspberry $\mathrm{Pi}$ controller which can monitor data and send it over remote receiver. So use of number of computers is reduced.

\section{References}

1. Alfredo Gardel Vicente, Ignacio Bravo Munoz Jose Luis Lazaro Galilea and Pedro A. Revenga del Toro, "Remote Automation Laboratory Using a Cluster of Virtual Machines," IEEE Transactions on Industrial Electronics, vol. 57, no. 10, pp. 3276-3283, 2010.

2. Amiya Ranjan Panda, Utpal Mandal and Hare Krishna Ratha, "Integrated Monitoring of Encoder Status Parameters and GUI based Remote Control 
Panel Using Lab view," IJCA., vol. 43, no. 3, pp. 21$26,2012$.

3. Arkadiusz Jestratjew and Andrzej Kwiecien, "Performance of HTTP Protocol in Networked Control Systems," IEEE Transaction on Industrial Informatics, vol. 9, no. 1, pp. 271-276, 2013.

4. Eva basada-Portas, Jose A. Luiesde la Torre, and Jesus M.de la Cruz,"Remote Control Laboratory Using EJS Applets and TwinCAT Programmable Logic Controllers," IEEE Transaction on Education, vol.56,no.2,pp.550-558,2013.
5. Md. Nasimuzzaman Chowdhury, Md. Shiblee Nooman and Srijon Sarker, "Access Control of Door and Home Security by Raspberry Pi through Internet," IJSER, vol. 4, issue. 11, pp550-558, 2013

6. Su Chunli and Zhao Xiangmei, "Comparison on Application of DCS and FCS," IEEE Conference on ICDMA, pp. 358-36

\section{How to cite this article:}

Sayali Gajanan Desai et al (2017) ' Global Industrial Process Monitoring Using Raspberry Pi Controller', International Journal of Current Advanced Research, 06(05), pp. 3623-3627.

DOI: http://dx.doi.org/10.24327/ijcar.2017.3627.0333 